\title{
Equilibrium structures of rapidly rotating stars with shellular rotation
}

\author{
Kotaro Fujisawa and Yu Yamamoto \\ Advanced Research Institute for Science and Engineering, Waseda University, 3-4-1 Okubo, \\ Shinjuku-ku, Tokyo 169-8555, Japan \\ email: fujisawa@heap.phys.waseda.ac.jp
}

\begin{abstract}
We have developed a new numerical method for obtaining self-consistent structures of rapidly rotating stars. We obtained self-consistent equilibrium structures of rapidly rotating massive stars with shellular rotation by using the method. These equilibrium structures might be useful for both evolution of rapidly rotating massive stars and progenitor models of core-collapse supernovae simulations.
\end{abstract}

Keywords. stars: evolution, stars: rotation

\section{Introduction}

One of the most fascinating challenge in the stellar astrophysics is understanding the structures of rapidly rotating stars and their evolution. Some massive stars have rapid rotations and their outer layers cannot be well described by 1D stellar evolution models. Multi-dimensional models are required to describe evolution of rapidly rotating stars.

\section{Method and result}

We have developed a new numerical method for obtaining self-consistent structures of rapidly rotating baroclinic stars (Fujisawa 2015) to investigate multi-dimensional stellar evolution models. We calculated rapidly rotating progenitor models with shellular rotation by using the method. A parametric progenitor model developed by Yamamoto \& Yamada (2016) is adopted in our calculations. The progenitor has three layers and its mass is $22 M_{\odot}$ in the case of the non-rotating model. The density distributions of our numerical results are displayed in Fig.1. The outer layers are deformed by their rapid rotations.
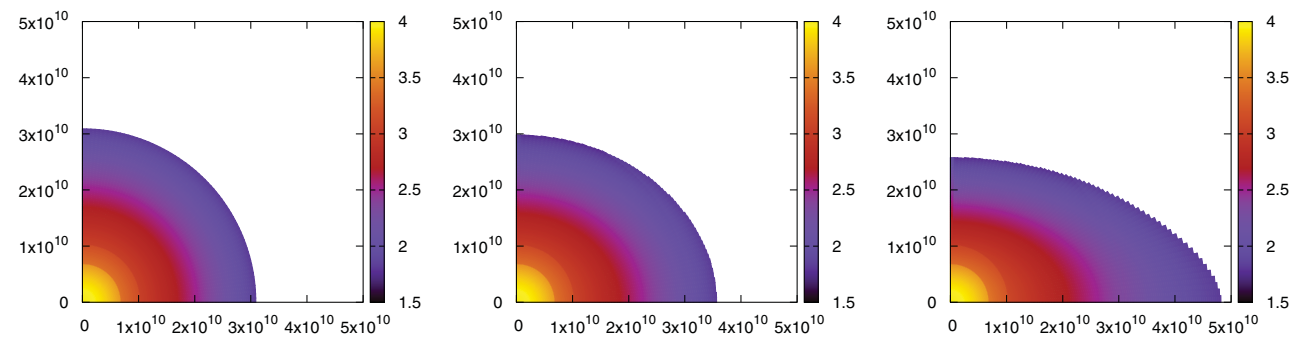

Figure 1. Density distributions $\left(\log _{10} \rho\right.$ in $\left[\mathrm{g} \mathrm{cm}^{-3}\right]$ unit) of the progenitor models. Left: Non-rotating model. Center: Rapid rotation model. Right: Extreme rotation model.

\section{References}

Fujisawa, K. 2015, MNRAS, 40, 1635

Yamamoto, Y. \& Yamada, S. 2016, ApJ, 818, 165 